\title{
The colourful world of quarks and gluons I: The shaping of Quantum Chromodynamics
}

\author{
Gerhard Ecker* \\ University of Vienna, Faculty of Physics \\ Boltzmanng. 5, A-1090 Wien, Austria \\ E-mail: gerhard.eckereunivie.ac.at
}

\begin{abstract}
In the first part of this public lecture, I recall the status of particle physics in the sixties of the last century before describing the phenomenon of scaling in deep inelastic lepton nucleon scattering that led to the discovery of asymptotic freedom. Within less than 15 years, quantum field theory arose like a phoenix from the ashes to provide a common framework for the gauge theories of electromagnetic, weak and strong interactions.
\end{abstract}

Xth Quark Confinement and the Hadron Spectrum,

October 8-12, 2012

TUM Campus Garching, Munich, Germany

${ }^{*}$ Speaker. 


\section{Introduction}

When Nora Brambilla asked me to deliver a public lecture on QCD, I suggested to split the lecture in two parts. In the first part, I briefly recall the status of particle physics in the sixties of the last century before describing the phenomenon of scaling in deep inelastic lepton nucleon scattering that led to the discovery of asymptotic freedom in 1973. In the second part, Thomas Mannel will discuss some of the many facets of QCD.

Why should one want to look back 50 years to understand the emergence of Quantum Chromodynamics, the nonabelian gauge theory of the strong interactions? Some of the motivations are:

- Particle physics in the early sixties looked very much different from now. In particular, there was no sign of anything like today's Standard Model.

- The strong interactions (the strong nuclear force as it was then usually called) were especially enigmatic, seemingly a hopeless case for quantum field theory. This was a serious deficiency because quantum field theory is the natural union of quantum theory and special relativity, two essential ingredients of particle physics.

- Within less than 15 years, quantum field theory arose like the famous phoenix from the ashes to provide the common framework for electromagnetic, weak and strong interactions.

- As a final motivation for my younger colleagues: whenever you feel depressed over the lack of "New Physics" at the LHC, sit back and recall the fascinating developments that led to the Standard Model in its present form.

As the audience was expected to consist not only of particle physicists, I have made an effort to keep the formalism to an absolute minimum.

\section{Particle physics in the sixties}

Already in those dark ages, it was known that all phenomena of the physical world can be traced back to only four fundamental forces:

\begin{tabular}{cc}
$\begin{array}{c}\text { gravitation } \\
\text { electromagnetism }\end{array}$ & $\begin{array}{c}\text { strong nuclear force } \\
\text { weak nuclear force }\end{array}$ \\
\hline long range & short range
\end{tabular}

Of the three interactions relevant at nuclear scales, electromagnetism was understood best. It was described by a bona fide quantum field theory called quantum electrodynamics (QED). Quantum corrections were calculable to any desired order in the fine-structure constant (at least in principle) and the theoretical predictions agreed with experiment. However, the actual treatment of the perturbative expansion (renormalization) was not generally accepted. Some of our forefathers (among them Dirac and Wigner) criticized the methodology as a mere recipe to "sweep the infinities 
under the rug". Even Feynman, one of the most influential particle physicists of the time, conceded [1] : "I do not subscribe to the philosophy of renormalization".

The weak interactions were described by the $V-A$ Fermi theory that accounted for the main features of weak decays. However, quantum corrections could not be calculated because the Fermi theory is the prototype of a nonrenormalizable quantum field theory. Towards the end of the decade, the renormalizable gauge field theory of electroweak interactions emerged [2].

Finally, as already emphasized, the strong interactions seemed to be a hopeless case for quantum field theory, for at least two reasons. For one, as more and more strongly interacting particles and resonances (hadrons) were found experimentally, it became less and less clear which should be the fundamental degrees of freedom to be described by quantum fields: nucleons (proton and neutron), pions, kaons, ... ? In addition, the strength of the interaction (the denomination "strong interactions" is after all not an accident) seemed prohibitive for a perturbative treatment, the only systematic method for treating quantum field theories known at the time. The general feeling among particle physicists in the early sixties was expressed by Landau [3]:

It is well known that theoretical physics is at present almost helpless in dealing with the problem of strong interactions. We are driven to the conclusion that the Hamiltonian method for strong interactions is dead and must be buried, although of course with deserved honour.

In the same spirit, Goldberger [4] formulated the general assessment of the relevance of quantum field theory:

My own feeling is that we have learned a great deal from field theory ... that I am quite happy to discard it as an old, but rather friendly, mistress who I would be willing to recognize on the street if I should encounter her again.

As quantum field theory appeared to be inapplicable for the strong interactions, other approaches were investigated, mainly along the following two lines.

\section{i. Bootstrap}

The bootstrap or nuclear democracy declared all hadrons to be equal. Instead of quantum field theories, only experimentally accessible observables should be investigated, using general properties of the S-matrix such as unitarity and analyticity [5]. By definition, the method was politically correct but not predictive: the full power of quantum field theory was lacking.

ii. Symmetries

From regularities in hadronic spectra and from relations between different hadronic processes, (approximate) symmetries were postulated and their consequences investigated. In addition to the well-established isospin symmetry, the group $S U(3)$ was introduced by GellMann and Ne'eman [6] to classify the observed hadrons and to predict the existence of states yet to be detected.

The symmetry approach gave insight into the world of hadrons, but an important question remained: how does one actually calculate hadronic processes, if quantum field theory was unreliable? The most influential approach during much of the sixties was put forward by Gell-Mann who suggested to use field theoretic toy models with the proper symmetries to abstract algebraic relations that could then be exploited independently of the underlying (and probably unreliable) field theories. The method became to be known as the "French cuisine approach" [7]: 


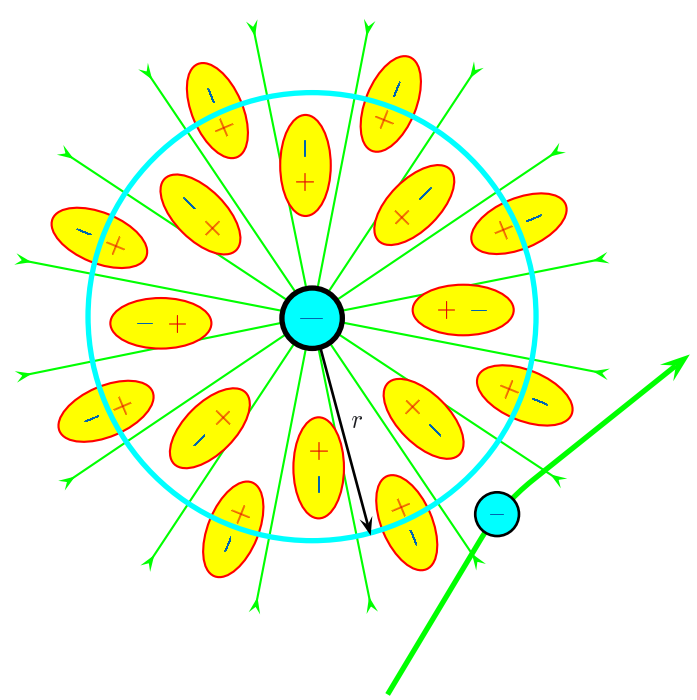

Figure 1: Electron electron scattering and vacuum polarization.

We construct a mathematical theory of the strongly interacting particles, which may or may not have anything to do with reality, find suitable algebraic relations that hold in the model, postulate their validity, and then throw away the model. We may compare this process to a method sometimes employed in French cuisine: a piece of pheasant meat is cooked between two slices of veal, which are then discarded.

\section{Scaling and asymptotic freedom}

In the late sixties, a decisive step forward in our understanding of the strong interactions came from experiment. The MIT-SLAC collaboration [8] investigated the so-called deep inelastic scattering of electrons on protons $e^{-}+p \rightarrow e^{-}+X$ where the hadronic debris $X$ was measured as a whole (inclusive measurement). The results were unambiguous and unexpected:

- Whereas at low energies the cross-section for electron proton scattering exhibits a lot of structure, it becomes increasingly structureless at high energies (and large momentum transfer): in simple terms, the phenomenon of "scaling" suggested that nucleons consisted of non-interacting part(on)s at high energies.

- Obvious candidates for the partons were the quarks that had previously been postulated as hadron constituents [9].

- However, this gave rise to a seeming paradox: how could the quarks be quasi-free at high energies and yet be permanently bound in hadrons, as free quarks have never been observed in isolation?

Expressing the puzzle in different words, the strength of the strong interactions seemed to be energy-dependent. Rather than a puzzle, this was actually a well-known effect in QED, with an 
important difference however: in contrast to the strong interactions, the strength of the electromagnetic interaction increases with energy. This phenomenon of "charge screening" is of quantum field theoretical origin: the space around an electron (or any charged particle) is populated by virtual electron-positron pairs that modify the effective charge of the electron felt by a another electron as shown in Fig.1. As the two electrons get closer to each other, the effective charge increases at short distances. In quantum theory short distances are tantamount to high energies and therefore the behaviour of the effective charge is opposite to what is suggested by scaling for the strong interactions.

In quantum field theory, the behaviour of the effective charge is governed by the $\beta$-function,

$$
\beta\left(q_{\mathrm{eff}}\right) \sim-r \frac{d q_{\mathrm{eff}}(r)}{d r}>0
$$

where $r$ is the distance from the bare charge. In contrast to QED, the $\beta$-function for the strong coupling constant (the analogue of the charge in QED) must therefore be negative to explain scaling. If the negative sign prevails to highest energies, the coupling strength vanishes asymptotically and the theory is called asymptotically free.

Once a plausible explanation of scaling was identified, the task remained to find an asymptotically free quantum field theory for the strong interactions. This turned out to be highly nontrivial: most of the (renormalizable) theories exhibited screening just like QED. The majority view in early 1973 was expressed by Zee [10]:

We conjecture that there are no asymptotically free quantum field theories in four dimensions.

While Coleman and Gross [11] were working on a general proof of this conjecture, their graduate students Politzer and Wilczek attempted to close a "loophole": the $\beta$-function for nonabelian gauge theories (Yang-Mills theories) was still unknown or at least unpublished ${ }^{1}$. In two successive papers in the Physical Review Letters in the spring of 1973 [13], the asymptotic freedom of YangMills theories was demonstrated. For most workers in the field, this was the explanation of scaling. David Gross himself put it this way [14]:

Like an atheist who has just received a message from a burning bush, I became an immediate true believer.

What is then the determining factor why nonabelian gauge field theories are asymptotically free in contrast to the abelian QED? In short, photons are electrically neutral, whereas the quanta of the strong interactions (to be called gluons) carry "colour" charge. Moreover, this can only work if, unlike the single photon, there is more than one type of gluons.

\section{Quantum Chromodynamics}

Although for most particle physicists the discovery of asymptotic freedom marks the birth of Quantum Chromodynamics, all ingredients of the theory were already "known" in 1972.

- The additional degree of freedom called colour was well established: quarks come in three colours [15].

\footnotetext{
${ }^{1}$ See Ref. [12] for an account of the earlier history of the subject.
} 
- Unknown to many, the existence of eight gluons was already proposed in 1972. By whom?

In the early seventies, Fritzsch and Gell-Mann were investigating (in the spirit of the French cuisine approach) a toy model with coloured quarks, but with a single gluon only. That such a model with a single gluon (necessarily not asymptotically free!) was considered for an investigation of scaling, gives evidence to the low prestige of perturbative quantum field theory for the strong interactions. In their contribution to the 1972 High Energy Physics Conference in Chicago, one can find an interesting passage [16]:

Now the interesting question has been raised lately whether we should regard the gluons as well as the quarks as being non-singlets with respect to color [5].

$\cdots$

Ref. [5]: J. Wess (private communication to B. Zumino)

For the non-experts: even if you don't know anything about group theory (the colour group $S U(3)$ in this case), you will accept that

$$
\overline{3} \times 3=1+8 .
$$

This equation tells us that if gluons are to couple to quarks at all, which they must in order to mediate the strong interactions, there are only two possibilities: either one or eight gluons. In 2005 I had the opportunity to ask Julius Wess about his suggestion. For him it seemed obvious, if the confinement of quarks had anything to do with the group structure of $S U(3)$, confinement of the gluons could only be understood if they strongly interact among themselves (in technical terms: they should carry colour like the quarks). I also asked him why he did not pursue this hypothesis at the time, but this is another story.

The issue of the number of gluons was finally and definitely settled in favour of the octet picture in the fall of 1973 [17].

The name Quantum Chromodynamics is generally attributed to Gell-Mann. It is less known where the name first appeared in printed form. The earliest appearance I could find is in a paper of Fritzsch, Gell-Mann and Minkowski [18] where they write in a footnote:

A good name for this theory is Quantum Chromodynamics.

When particle theorists discuss about a quantum field theory, they do so mainly in terms of the associated Lagrangian (rather than the field equations). Without explaining any details, I close this first part of the lecture by exposing the Lagrangians for QED and QCD to show the similarities between these gauge field theories. Restricting the electromagnetic interaction to electrons and photons, the Lagrangian of QED takes the form

$$
\mathscr{L}_{\mathrm{QED}}=\bar{\psi}(i \not D-m) \psi-\frac{1}{4} F_{\mu v} F^{\mu v}
$$

where $\psi$ stands for the electron field, $m$ is its mass and the electromagnetic field strength tensor $F_{\mu \nu}$ already appears in the classical Maxwell equations. The covariant derivative $\not D$ contains the photon field. For a single type (flavour) of quarks, the QCD Lagrangian looks very similar:

$$
\mathscr{L}_{\mathrm{QCD}}=\sum_{i, j=1}^{3} \bar{q}_{i}\left(i \not D-m_{q}\right)_{i j} q_{j}-\frac{1}{4} \sum_{\alpha=1}^{8} G_{\mu \nu}^{\alpha} G^{\alpha, \mu v} .
$$




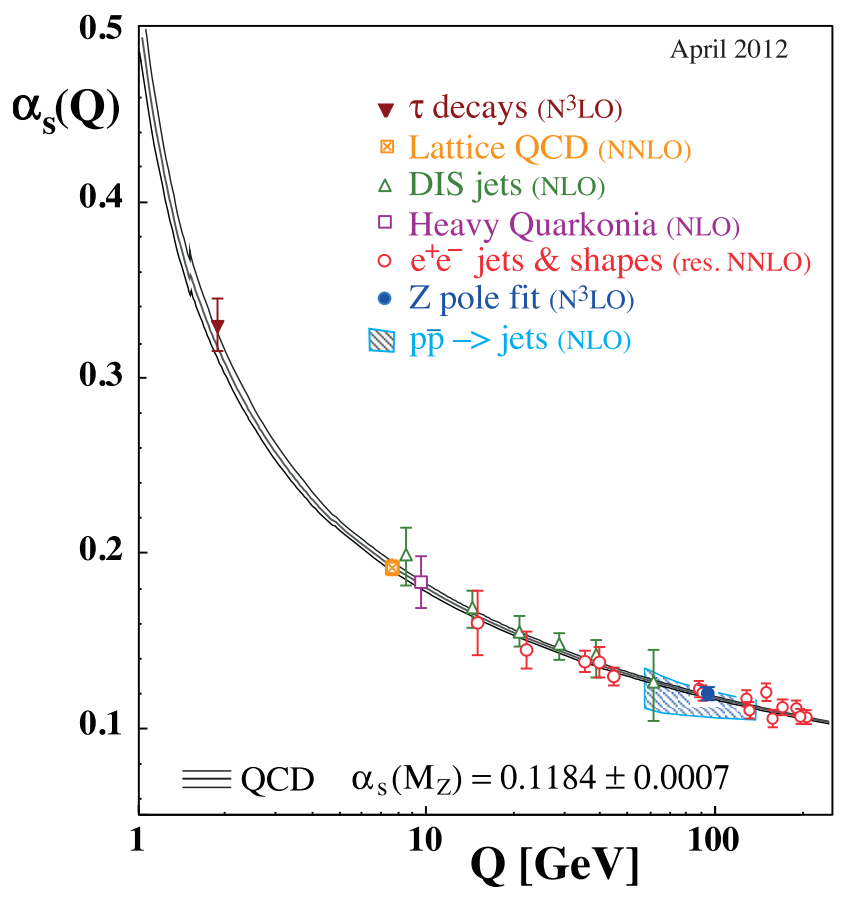

Figure 2: Energy dependence of the "strong fine-structure constant" $\alpha_{s}(Q)$ from various sources [19]. Also shown is the impressive agreement with the QCD prediction.

The quark fields $q_{i}$ carry a colour index $(i=1,2,3)$ and there are eight gluon field strength tensors $G_{\mu \nu}^{\alpha}$. All the difference between QED and QCD resides in $G_{\mu \nu}^{\alpha}$ that, compared to $F_{\mu \nu}$, depends in a more complicated way on the gluon field. In fact, this makes a lot of difference (nonabelian vs. abelian gauge theory): QCD is a much richer quantum field theory than QED. In particular, this difference is responsible for asymptotic freedom. The present status of the energy dependence of the interaction strength is displayed in Fig. 2.

Some of the many facets of this fascinating theory will now be presented by Thomas Mannel.

\section{References}

[1] R. P. Feynman, in The Quantum Theory of Fields - The $12^{\text {th }}$ Solvay Conference, Interscience Publisher, New York 1961.

[2] S. L. Glashow, Partial symmetries of weak interactions, Nucl. Phys. 22 (1961) 579;

S. Weinberg, A model of leptons, Phys. Rev. Lett. 19 (1967) 1264;

A. Salam, Weak and electromagnetic interactions, in Proceedings of the $8^{\text {th }}$ Nobel Symposium, Almqvist and Wiksell, Stockholm 1968.

[3] L. D. Landau, Fundamental problems, in The Pauli Memorial Volume, Interscience Publisher, New York 1960. 
[4] M. L. Goldberger, in The Quantum Theory of Fields - The $12^{\text {th }}$ Solvay Conference, Interscience Publisher, New York 1961.

[5] G. F. Chew, The Analytic S-Matrix: a Basis for Nuclear Democracy, W.A. Benjamin Inc., New York 1966.

[6] M. Gell-Mann, The eightfold way: a theory of strong interaction symmetry, CIT Synchrotron Laboratory Report CTSL-20 (1961);

Y. Ne'eman, Derivation of strong interactions from a gauge invariance, Nucl. Phys. 26 (1961) 222.

[7] M. Gell-Mann, The symmetry group of vector and axial vector currents, Physics 1 (1964) 63.

[8] M. Breidenbach et al., Observed behavior of highly inelastic electron-proton scattering, Phys. Rev. Lett. 23 (1969) 935.

[9] M. Gell-Mann, A schematic model of baryons and mesons, Phys. Lett. 8 (1964) 214;

G. Zweig, An SU(3) model for strong interaction symmetry and its breaking, in Developments in the Quark Theory of Hadrons, D.B. Lichtenberg and S.P. Rosen, eds., CERN Geneva - TH. 401 (1964).

[10] A. Zee, Study of the renormalization group for small coupling constants, Phys. Rev. D 7 (1973) 3630.

[11] S. Coleman and D. J. Gross, Price of asymptotic freedom, Phys. Rev. Lett. 31 (1973) 851.

[12] G. 't Hooft, When was asymptotic freedom discovered? Or the rehabilitation of quantum field theory, Nucl. Phys. Proc. Suppl. 74 (1999) 413 [hep-th/9808154].

[13] D. J. Gross and F. Wilczek, Ultraviolet behavior of nonabelian gauge theories, Phys. Rev. Lett. 30 (1973) 1343;

H. D. Politzer, Reliable perturbative results for strong interactions?, Phys. Rev. Lett. 30 (1973) 1346.

[14] D. J. Gross, Twenty five years of asymptotic freedom, Nucl. Phys. Proc. Suppl. 74 (1999) 426 [hep-th/9809060].

[15] W. A. Bardeen, H. Fritzsch and M. Gell-Mann, Light cone current algebra, $\pi^{0}$ decay, and $e^{+} e^{-}$ annihilation, CERN-TH-1538 (1972) and earlier references therein.

[16] H. Fritzsch and M. Gell-Mann, Current algebra: quarks and what else?, eConf C 720906 V2 (1972) 135 [hep-ph/0208010].

[17] H. Fritzsch, M. Gell-Mann and H. Leutwyler, Advantages of the color octet gluon picture, Phys. Lett. B 47 (1973) 365.

[18] H. Fritzsch, M. Gell-Mann and P. Minkowski, Vector - like weak currents and new elementary fermions, Phys. Lett. B 59 (1975) 256.

[19] J. Beringer et al. [Particle Data Group Collaboration], Review of Particle Physics (RPP), Phys. Rev. D 86 (2012) 010001. 